\title{
Development of a novel plant activator for rice diseases, tiadinil*
}

\author{
Kenji Tsubata, ${ }^{* *}$ Kiyoshi Kuroda, Yoshinobu Yamamoto ${ }^{\dagger}$ and Noriaki Yasokawa \\ Research Center, Nihon Nohyaku Co., Ltd., 345 Oyamada-Cho, Kawachi-Nagano, Osaka 586-0094, Japan \\ ${ }^{\dagger} R \&$ D Strategy Department, Research \& Development Division, Nihon Nohyaku Co. Ltd., \\ 1-2-5 Nihonbashi, Chuo-Ku, Tokyo 103-8236, Japan
}

(Accepted March 5, 2006)

\begin{abstract}
Tiadinil is a novel systemic fungicide for rice diseases discovered and developed by Nihon Nohyaku Co., Ltd. The compound is characterized by a unique heterocyclic moiety, 1,2,3-thiadiazole, and a unique mode of action of inducing the plant defense mechanism against a pathogen. The lead compound was found in the synthesis research of 1,2,3-thiadiazole chemistry with the appropriate screening system. Both application methods, nursery box and into-water applications, can be used in rice cultivation. It shows excellent activity against rice blast and also exhibits activity against some rice bacterial diseases. Tiadinil has a favorable toxicological, eco-toxicological and kinetic profile. (C) Pesticide Science Sociey of Japan
\end{abstract}

Keywords: tiadinil, plant activator, rice blast fungicide, 1,2,3-thiadiazole, nursery box application, into-water application.

\section{Introduction}

A novel class fungicide, tiadinil, acting as a plant activator, was discovered and developed by Nihon Nohyaku Co., Ltd. The characteristic of the chemical structure is a heterocyclic moiety, 1,2,3-thiadiazole. It can be used by both nursery box and into-water applications. From the mode of action of inducing plant resistance, tiadinil is considered to have a low risk of developing fungicide resistance. It was registered in Japan in 2003 (trade name: V-GET ${ }^{\circledR}$ ) and also approved in combination with some insecticides, fungicides, and herbicides.

This paper describes the history of the discovery, synthesis, structure-activity relationships, biological activity, safety, and mode of action of tiadinil.

\section{Discovery and Synthesis}

From an initial interest in the mode of action and the chemical structure, as well as information about benzo-1,2,3-thiadiazoles, we started research into new plant activator using 1,2,3thiadiazole chemistry. From the various 1,2,3-thiadiazole derivatives we synthesized, we found that only 1,2,3-thiadiazole5 -carboxylic acid derivatives showed high fungicidal activity

* See Part II for the full Japanese article.

** To whom correspondence should be addressed. (C) Pesticide Science Society of Japan against rice blast. After optimization of the derivatives to find a compound which can be used both for nursery box and intowater applications, we selected tiadinil as a development candidate considering various factors such as a balance between quick-acting and long-lasting efficacy, phytotoxicity, water solubility, convenience of formulation, formulation stability, production cost, and safety.

We investigated the synthesis of tiadinil and found three synthetic routes: two start from methyl acetoacetate and the other starts from diketene.

\section{Structure-Activity Relationships}

Among the 1,2,3-thiadiazole derivatives we synthesized only one compound, 4-ethyl-5-methoxycarbonyl derivative, showed good blasticidal activity by into-water application. A carboxy group at 5-position was considered important. As a substituent at the 4-position of 1,2,3-thiadiazole-5-carboxylic acid esters, a substituent with a chain length of one to three is optimal, and hydrophobicity is also an important factor in the activity. Concerning substituents at the 5-position of 4methyl-1,2,3-thiadiazole-5-carboxylic acid derivatives, esters and anilides showed high activity in general, while aliphatic amides and $N$-methylanilide showed low activity. It is suggested that the generation of 5-carboxylic acid is important by hydrolysis or oxidation. 3'-Chloro-4'-methylanilide showed very good activity under different conditions throughout the selection process of the development candidate. Appropriate 
solubility in water and hydrolyzability seemed important.

\section{Physical and Chemical Properties}

Common name: tiadinil

Trade name: V-GET ${ }^{\circledR}$

Experimental name: NNF-9850

CAS registry No. :223580-51-6

Chemical name: 3'-Chloro-4,4'-dimethyl-1,2,3-thiadiazole-5carboxanilide

Molecular formula: $\mathrm{C}_{11} \mathrm{H}_{10} \mathrm{ClN}_{3} \mathrm{OS}$

Molecular weight: 267.74

Appearance: pale yellow solid

Melting point: $112.2^{\circ} \mathrm{C}$

Solubility in water: $13.2 \mathrm{mg} / 1\left(20^{\circ} \mathrm{C}\right)$

Vapor pressure: $1.06 \times 10^{-6} \mathrm{~Pa}\left(25^{\circ} \mathrm{C}\right)$

Partition coefficient: $\log P_{\mathrm{OW}}=3.68\left(25^{\circ} \mathrm{C}\right)$

\section{Biological Activity}

In field trials, tiadinil exhibited excellent activity against rice blast by nursery box application at a dosage of $6 \mathrm{~g}$ a.i./nursery box, and the control activity lasted more than 80 days after treatment. When tiadinil was applied at 7 20 days before the first appearance of leaf blast by into-water application, tiadinil also showed stable control activity at a dosage of $1800 \mathrm{~g}$ a.i./ha. The control activity of tiadinil was not affected under various field conditions such as infection pressure of disease, kinds of soil, depth of paddy water, depth of transplanted seedlings and so on. Tiadinil could be used in rice cultivating areas where previously developed resistant strains against conventional products have been occupied. Tiadinil exhibited additional control activity against some bacterial diseases such as rice bacterial leaf blight and grain rot in field trials, which suggested that tiadinil was adaptable to the simultaneous control of a wide range of rice diseases in practical use.

\section{Safety}

Tiadinil and its formulation exhibited quite low acute toxicity toward laboratory animals via any exposure route. No critical eye or skin irritation, or skin sensitization was recognized. Prolonged exposure studies demonstrated that tiadinil exhibited no evidence of carcinogenicity, teratogenicity, and reproduction toxicity. The toxicity of tiadinil toward non-target aqueous species (fish, crustacean, and algae) earthworms, silk worms, and honey bees was also quite low. Metabolism studies employing radiolabeled compounds showed that the compound was rapidly metabolized via hydrolysis of the amide bond and oxidation of the methyl moiety (4-position of thiadiazole ring and phenyl ring) in plants, soil and animals. Mineralization (evolution of carbon dioxide) was also found in soil and animal metabolism. No significant translocation of tiadinil and its metabolites into rice grain was found.

\section{Mode of Action}

Using the cellophane film method, tiadinil did not inhibit the morphogenesis of the infection process of rice blast fungus, Magnaporthe grisea, although the intracellular hyphal growth of $M$. grisea was remarkably inhibited in the first invaded cell of the inner epidermal tissues of rice leaf sheath excised from tiadinil-treated rice plants. In the invaded cells, the deposition of callose was observed both microscopically and cytochemically. The phenomena, including the restriction of hyphal development and the cytoplasmic reaction of invaded cells, were similar to those of resistant reactions in the incompatible combination of rice cultivar and fungus under natural conditions. Moreover, the enhancement of several resistant-related genes reported previously such as $P B Z 1, R P R-1$ and $P A L-Z B 8$ was detected in tiadinil-treated rice plants. These results suggested strongly that tiadinil provided excellent blast control by activation of the resistance mechanism possessed primarily in the host plant, and worked as a plant activator in rice. 